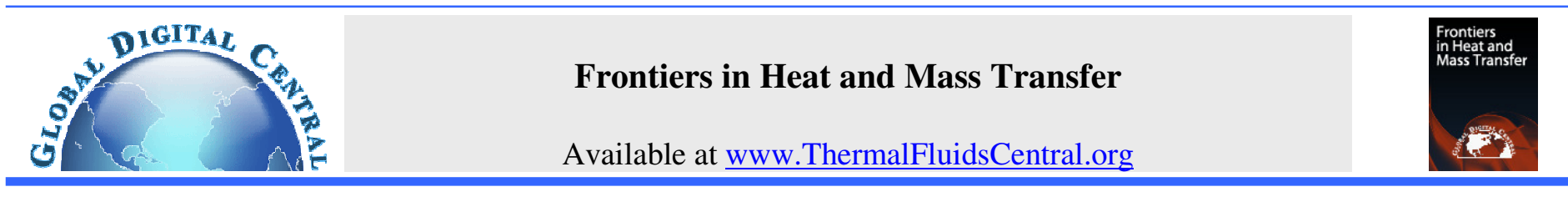

\title{
MULTIPLE SLIPS AND CHEMICAL REACTION EFFECTS ON MHD STAGNATION POINT FLOW OF CASSON FLUID OVER A STRETCHING SHEET WITH VISCOUS AND JOULES HEATING
}

\author{
G. Vinod Kumar, R. V. M. S. S. Kiran Kumar*, S. V. K. Varma \\ Department of Mathematics, S. V. University, Tirupati, 517502, A.P., India.
}

\begin{abstract}
The steady boundary layer stagnation flow of a Casson fluid over a stretching sheet with slips boundary conditions in the presence of viscous dissipation, Joule heating and the first order destructive chemical reaction is analyzed. The governing flow problem is based on momentum equation, energy equation, and mass diffusion equation and these are further simplified with the help of similarity transformations. The reduced, resulting highly nonlinear coupled ordinary differential equations are solved using the Matlab bvp4c package. The effects of various parameters on the dimensionless velocity, temperature, and concentration as well as on the skin friction coefficient and the rate of heat and mass transfer coefficients are presented in graphical forms. A comparison with published data has been carried out and good agreements are found, it is noted that the effects of increasing the value of the Casson parameter are to suppress the fluid velocity.
\end{abstract}

Keywords: Magnetohydrodynamics, Casson fluid, Stagnation point flow, Multiple slips, Joules heating.

\section{INTRODUCTION}

The flow near the stagnation point has made the attention of many investigators for more than a century because of its wide applications. Some of the applications are cooling of nuclear reactors during the emergency shutdown, cooling of electronic devices by fans, solar central receivers exposed to wind currents, and many hydrodynamic processes in engineering applications. The author Hiemenz (1911) originated the study of a stagnation point flow towards a solid surface in moving fluid. He was the pioneer to analyze two-dimensional stagnation point flow on the stationary plate to reduce the NavierStokes equations to nonlinear ordinary differential equations. Since then many investigators have extended the idea to a different aspect of the stagnation point flow problems. The study of hydrodynamic stagnation point flow over a stretching surface has attracted much attention due to their many practical applications such as MHD generators and cooling of infinite metallic plates in a bath. Hiemenz (1911) first studied twodimensional stagnation flow using similarity transformations to reduce the Navier-Stokes equations to nonlinear ordinary differential equations. Different aspects of the stagnation point flow had been considered by many researchers. The magnetohydrodynamic stagnation point flow over a stretching sheet with variable surface temperature is considered by Ishak et al. (2009). Ramesh et al. (2014) analyzed the magnetohydrodynamic stagnation point radiative dusty fluid flow towards a stretching sheet, and concluded that the fluid phase is higher than the dust phase. Al-sudais (2012) numerically investigate the effects of variable thermal conductivity and heat source/sink on MHD viscous fluid flow near a stagnation point on a non-conducting stretching sheet.

Many of the fluids that are used in industries shows nonNewtonian behavior, so the modern day investigators are showing keen interest in those industrial non-Newtonian fluids, and their dynamics. All the properties of such non-Newtonian fluids are not covered by a single constitutive equation and hence many non-Newtonian fluid models (Wilkinsion, (1970), Rajagopal, (1980), Rajagopal, et al. (1984) and Dorier and Tichy, (1992)) have been proposed to clarify all physical behaviors. Among them Casson fluid is one of the types of such non-Newtonian fluids, that behaves like an elastic solid, and for this a yield shear stress exists in the constitutive equation. Casson model is claimed to fit rheological data better than general viscoplastic models for many materials and also a preferred rheological model for blood and chocolate (see (Casson, (1959))). The Casson fluid flow in a pipe with a homogeneous porous medium was investigated by Dash et al. (1996). The Casson model fits the flow data better than the more general Herschel-Bulkley model by Joye (1998) and Kirsanov and Remizo (1999), which is a power-law formulation with yield stress as Bird et al. (1960). Bhattacharyya (2013) examined the stagnation point flow of Casson fluid towards a shrinking/stretching sheet. Hayat et al. (2012) considered the mixed convection stagnation point flow of an incompressible non-Newtonian fluid over a stretching sheet under convective boundary conditions. Ramesh (2016) investigated the stagnation-point flow of an incompressible non-Newtonian fluid over a non-isothermal stretching sheet. Animasaun et al. (2016) studied free convective MHD Casson fluid flow past an exponentially stretching sheet and concluded that rising values of variable plastic dynamic viscosity parameter of Casson fluid correspond to enhance the momentum boundary layer thickness. The magnetohydrodynamic heat transfer Casson fluid flow over a permeable stretching sheet in a porous medium with non-uniform heat generation/absorption and first order chemical reaction is considered by Gireesha et al (2015). Later, Nadeem et al. (2012) examined the MHD boundary layer flow of a Casson fluid over an exponentially penetrable shrinking sheet. Bhattacharyya (2013) considered the two-dimensional MHD stagnation point flow of electrically conducting non-Newtonian Casson fluid and heat transfer towards a stretching sheet. 
In the above-stated papers, the investigators did not consider the slip boundary conditions. In certain circumstances, the central idea for Navier-stokes theory is the no-slip condition. So, on applying the no slip boundary condition many authors have obtained both numerical and analytical solutions to study velocity and temperature profiles.

The no-slip condition is not enough for most non-Newtonian liquids, as some polymer melts often shows minuscule wall slip and that has a controlling influence by a non-linear and monotone relation between the slip velocity and the traction. Partial velocity slip may happen on the stretching boundary when the fluid is particulate such as emulsions, suspensions, foams and polymer solutions. In various industrial processes the slip effects can arise at the boundary of pipes, walls, curved surfaces, etc. Navier slip condition is a usual approach to study slip phenomena. The thermal and solutal slip conditions may also arise in many engineering developments. The boundary layer slip flow problems arise in improving of artificial heart valves and internal cavities. The MHD and slip effect in the boundary layer viscous flow over a surface with heat transfer was examined by Bhattacharyya et al. (2011). Nandy (2013) studied the hydromagnetic flow of a Casson fluid in the neighborhood of a stagnation point over a stretching surface in the presence of velocity and thermal slips. The multiple slips on magnetohydrodynamic Jeffrey nanofluid flow over a horizontal stretching surface in the presence of radiation has been considered by Prasanna kumara et al. (2016). The effects of viscous dissipation and Joule heating are usually characterized by the Eckert number and the product of the Eckert number with magnetic parameter respectively, and both effects are important in geophysical flows and in nuclear engineering (see Alim et al., (2007)). Joule heating is the process by which the passage of an electric current through a conductor produces heat. Joule heating is used in uncountable number of gadgets and industrial process such as electric stoves, electric heaters, soldering irons, electric fuses, and thermistors etc. Viscous dissipation changes the temperature distributions by playing a role like an energy source, which leads to affected heat transfer rates. The effect of viscous dissipation depends on whether the plate is being cooled or heated. Heat transfer analysis over a porous surface is of much practical interest due to its abundant applications. The earlier studies are based on the constant physical properties of the fluid. However, it is known that the significant change may occur in the physical properties of the fluid with temperature. The increase in temperature leads to the growth in the transport phenomena by decreasing the viscosity across the velocity boundary layer and due to which the heat transfer rate at the wall is also affected. Therefore, to accurately predict the flow and heat transfer rates, it is essential to take into account the temperature-dependent viscosity of the fluid.

Apart from the viscous dissipation in magnetohydrodynamic flows, the Joules dissipation also acts as a volumetric heat source. The problem of viscous dissipation, Joule heating and heat source/sink on non-Darcy magnetohydrodynamic natural convection flow over an isoflux permeable sphere in a porous medium is numerically analyzed by Yih (2000). The effect of viscous dissipation and Joule heating on magnetohydrodynamic free convection flow past a semi-infinite vertical flat plate in the presence of the Hall and non-slip current effects for the case of power-law variation of the wall temperature is analyzed by Abo-Eldahab and El Aziz (2005).The magnetohydrodynamic stagnation point flow of a Casson fluid over a nonlinearly stretching sheet with viscous dissipation is analyzed by Medikare et al. (2016). Turkyilmazoglu (2011) investigate the MHD slip flow and heat transfer effects with dissipative energy, thermal radiation and internal heat source/sink in conducting fluid flow over a porous stretching sheet.

The present study aims to provide a detailed study of the combined viscous and Joule dissipation effects on MHD stagnation point flow of an electrically conducting viscous incompressible Casson fluid through a permeable stretching sheet with multiple slips, thermal radiation, and first order chemical reaction.

\section{MATHEMATICAL FORMULATION}

A study two-dimensional laminar boundary layer MHD stagnation point flow of viscous incompressible electrically conducting Casson fluid bounded by stretching sheet at $y=0$ with slips boundary conditions in the presence of thermal radiation, first order chemical reaction and, viscous and Joules dissipation. The coordinate system chosen in such a way that the axis is along the sheet and y-axis is normal to the stretching sheet (see Fig.1). It is assumed that the velocity distribution far from the surface is given by $u=U(x)=a x$, where $a$ is

a positive constant. $T_{w}$ and $C_{w}$ are the uniform temperature and concentration at the stretching surface, respectively. $T_{\infty}$ and $C_{\infty}$ are the free stream temperature and concentration, respectively. The magnetic Reynolds number is assumed very small, and thus the included magnetic field is negligible. The model of first order chemical reaction is considered. The level of concentration of foreign mass assumes to be low, so that Soret and Dufour effects are negligible. It assumed that the flow is generated by stretching of non-conducting elastic bounding sheet from the employment of two equal and opposite forces along $\mathrm{x}-$ axis in such a way that the origin remains fixed and velocity of the boundary sheet is a linear order in the flow direction. In conjugate heat transfer flow problems thermal conductivity is dependent on the temperature (see Mohammad et al. (2014)), here it is assumed that the thermal conductivity is independent of temperature. It is also assumed the rheological equations of the state for an isotropic and incompressible flow of Casson fluid can be written as (Mustafa et al. (2012))

$$
\tau_{i j}=\left\{\begin{array}{l}
2\left(\mu_{\beta}+\rho_{y} / \sqrt{2 \pi}\right) e_{i j}, \pi>\pi_{c} \\
2\left(\mu_{\beta}+\rho_{y} / \sqrt{2 \pi_{c}}\right) e_{i j}, \pi<\pi_{c}
\end{array}\right.
$$

where $\tau_{i j}$ is the $(i, j)^{t h}$ component of the stress tensor, $\pi=e_{i j} e_{i j}, e_{i j}$ is the $(i, j)^{\text {th }}$ component of deformation rate, $\pi$ denotes the product of the component of deformation rate with itself, and $\rho_{y}$ is the yield stress of a fluid, $\pi_{c}$ is a critical value of this product based on the nonNewtonian model, $\mu_{B}$ is the plastic dynamic viscosity of the nonNewtonian fluid.

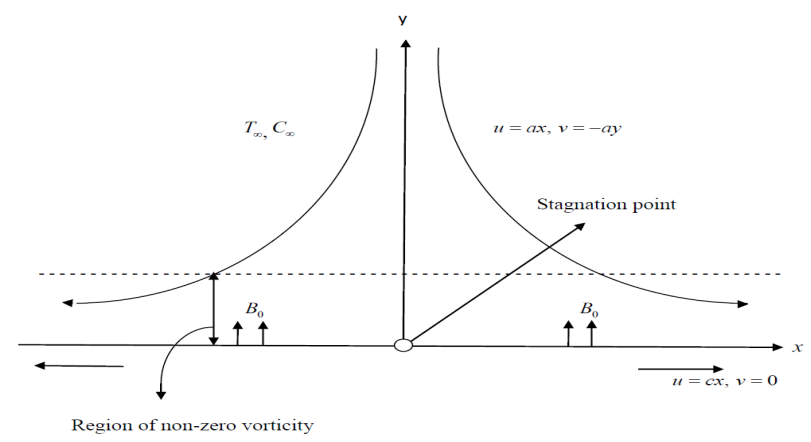

Fig. 1 Physical model and Co-ordinate system.

Under the previous assumptions, the MHD boundary layer equation for steady stagnation point flow can be written as

$$
\begin{aligned}
& \frac{\partial u}{\partial x}+\frac{\partial v}{\partial y}=0, \\
& u \frac{\partial u}{\partial x}+v \frac{\partial v}{\partial y}=U \frac{\partial U}{\partial x}+v\left(1+\frac{1}{\beta}\right) \frac{\partial^{2} u}{\partial y^{2}}+\frac{\sigma B_{0}{ }^{2}}{\rho}(U-u), \\
& u \frac{\partial T}{\partial x}+v \frac{\partial T}{\partial y}=\kappa \frac{\partial^{2} T}{\partial y^{2}}+\frac{v}{c_{p}}\left(1+\frac{1}{\beta}\right)\left(\frac{\partial u}{\partial y}\right)^{2}+\frac{\sigma B_{0}{ }^{2}}{\rho c_{p}}(U-u)^{2}-\frac{1}{\rho c_{p}} \frac{\partial q_{r}}{\partial y},
\end{aligned}
$$


$u \frac{\partial C}{\partial x}+v \frac{\partial C}{\partial y}=D_{m} \frac{\partial^{2} C}{\partial y^{2}}-k_{l}\left(C-C_{\infty}\right)$,

where $x$ and $y$ represents coordinate axis along the continuous surface in the direction of motion and normal to it respectively. The velocity components along $x$ and $y$ axis are $u$ and $v$ respectively. $\sigma$ is the electrical conductivity, $\rho$ is the density of the fluid, $B_{0}$ is the uniform magnetic field strength, $\kappa$ is thermal diffusivity, $c_{p}$ is specific heat at constant pressure, $v$ is the kinematic viscosity, $q_{r}$ is the radiative heat flux, $D_{m}$ is the molecular diffusivity, $k_{l}$ is the dimensional chemical reaction parameter, and $\beta$ is the Casson parameter.

Using the Rosseland approximation for radiation we have,

$q_{r}=-\frac{4 \sigma^{*}}{3 k^{*}} \frac{\partial T^{4}}{\partial y}$

where $\sigma^{*}$ is the Stefan-Boltzmann constant and $k_{l}$ is the absorption coefficient. Assuming that the temperature differences within the flow such that the term $T^{4}$ may be expanded in Taylor series as Brewster (1972)

$T^{4}=4 T_{\infty}^{3} T-3 T_{\infty}^{4}$

In view of eqns. (6) and (7) the eqn. (4) can be written as

$u \frac{\partial T}{\partial x}+v \frac{\partial T}{\partial y}=\kappa \frac{\partial^{2} T}{\partial y^{2}}+\frac{v}{c_{p}}\left(1+\frac{1}{\beta}\right)\left(\frac{\partial u}{\partial y}\right)^{2}+\frac{\sigma B_{0}{ }^{2}}{\rho c_{p}}(U-u)^{2}+\frac{16 \sigma^{*} T_{\infty}^{3}}{3 \rho c_{p} k^{*}} \frac{\partial^{2} T}{\partial y^{2}}$,

The corresponding slip boundary conditions are

$u=c x+L \frac{\partial u}{\partial y}, v=0, T=T_{w}+S \frac{\partial T}{\partial y}, C=C_{w}+D \frac{\partial C}{\partial y}$ at $y=0$,

$u \rightarrow U(x)=a x, T \rightarrow T_{\infty}, C \rightarrow C_{\infty}$ as $y \rightarrow \infty$

Where $L$ is the velocity slip factor, $S$ is the thermal slip factor, $D$ is the solutal slip factor and $a, c$ are positive constants.

\section{METHOD OF SOLUTION}

The momentum and energy equations can be transformed into the corresponding nonlinear ordinary differential equations by the following transformation:

$\psi=x \sqrt{c v} f(\eta), \theta(\eta)=\left(\frac{T-T_{\infty}}{T_{w}-T_{\infty}}\right), \phi(\eta)=\left(\frac{C-C_{\infty}}{C_{w}-C_{\infty}}\right), \eta=y \sqrt{\frac{c}{v}}$

The continuity equation can be satisfied by introducing a stream function $\psi$ such that

$u=\frac{\partial \psi}{\partial y}, v=-\frac{\partial \psi}{\partial x}$

where $\eta$ is independent similarity variable and $T_{w}=T_{\infty}+b x^{2}, \mathrm{~b}$ is a positive constant. Using the above relations in Eqns. (3), (5) and (8), we get the transformed nonlinear ordinary differential equations as

$\left(1+\frac{1}{\beta}\right) f^{\prime \prime \prime}+f f^{\prime \prime}-f^{\prime 2}+\alpha^{2}+M\left(\alpha-f^{\prime}\right)=0$,

$\frac{1}{\operatorname{Pr}}\left(1+\frac{4 R}{3}\right) \theta^{\prime \prime}+f \theta^{\prime}-2 f^{\prime} \theta+\left(1+\frac{1}{\beta}\right) E c f^{\prime \prime 2}+M E c\left(\alpha-f^{\prime}\right)^{2}=0$,

$\frac{1}{S c} \phi^{\prime \prime}+f \phi^{\prime}-K r \phi=0$.

Here $\alpha(=a / c)$ is the dimensionless velocity ratio parameter, $M\left(=\sigma \beta_{0} / \rho c\right)$ is the magnetic parameter, $\operatorname{Pr}=(v / \kappa)$ is the Prandtl number, $E_{c}=\left(c^{2} / b c_{p}\right)$ is Eckert number, $R=\frac{4 \sigma^{*} T_{\infty}^{3}}{k k^{*}}$ is the thermal radiation parameter, $S c=\frac{v}{D_{m}}$ is the Schmidt number, the chemical reaction parameter $K r=\frac{K_{l}}{c}$.

The transformed boundary conditions are

$f(0)=0, f^{\prime}(0)=1+\delta f^{\prime \prime}(0), \theta(0)=1+\gamma \theta^{\prime}(0), \phi(0)=1+\chi \phi^{\prime}(0)$

$f^{\prime}(\infty)=\alpha, \theta(\infty)=0, \phi(\infty)=0$

where $\delta=\sqrt{c / v} L$ is the dimensionless velocity slip parameter, $\gamma=\sqrt{c / v} S$ is the dimensionless thermal slip parameter, $\chi=\sqrt{c / v} D$ is the dimensionless solutal slip parameter.

The physical quantities of interest are skin friction coefficient $C_{f}$ and local Nusselt number $N u_{x}$, which are given by

$C_{f}=\frac{\tau_{w}}{\rho u_{w}{ }^{2}(x)}, N u_{x}=\frac{x q_{w}}{k\left(T_{w}-T_{\infty}\right)}, S h_{x}=\frac{x q_{m}}{k\left(C_{w}-C_{\infty}\right)}$,

Where $\tau_{w}$ is shear stress along the stretching surface and $q_{w}$ is the heat flux from the stretching surface, which are given by

$\tau_{w}=\left(k_{c}+\frac{\tau_{0}}{\sqrt{2 \pi_{c}}}\right)\left(\frac{\partial u}{\partial y}\right)_{y=0}, q_{w}=-k\left(\frac{\partial T}{\partial y}\right)_{y=0}$ and $q_{m}=-D_{m}\left[\frac{\partial C}{\partial y}\right]$

In the view of boundary conditions (9), we get

$\operatorname{Re}_{x}^{1 / 2} C_{f}=\left(1+\frac{1}{\beta}\right) f^{\prime \prime}(0), \frac{N u_{x}}{\operatorname{Re}_{x}^{1 / 2}}=-\theta^{\prime}(0)$ and $\frac{S h_{x}}{\operatorname{Re}_{x}^{1 / 2}}=-\phi^{\prime}(0)$

Where $\operatorname{Re}_{x}=u_{w}(x) x / v$ is a local Reynolds number.

\section{RESULTS AND DISCUSSION}

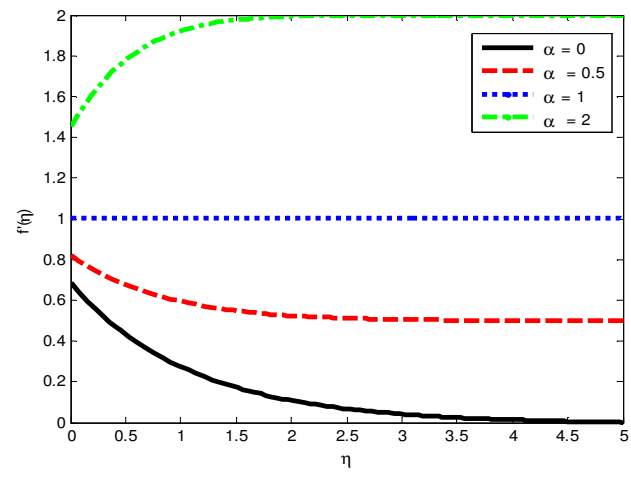

Fig. 2 Effect of $\alpha$ on $f^{\prime}(\eta)$.

The set of nonlinear coupled ordinary differential Eqs. (12) - (14) subject to the boundary conditions (15) are solved using Matlab bvp4c package. The computations are carried out until we get the velocity, temperature and concentration profiles converge and satisfy the far field boundary conditions (15) asymptotically. The numerical calculations were carried out for different values of the velocity ratio parameter $\alpha$, velocity slip parameter $\delta$, the magnetic field parameter $M$, Eckert number $E c$, thermal slip parameter $\gamma$, Casson parameter $\beta$, solutal slip parameter $\chi$, Radiation parameter $R$, Prandtl 
number $\operatorname{Pr}$, Chemical reaction parameter $K r$, Schmidt number $S c$, and their effects on the flow and heat and mass transfer characteristics are analyzed and discussed through graphs. Table-1 showed the accuracy of the method used and verified with the existing results, and they are found to be in good agreement with Mahapatra and Gupta (2002), Ishak et al. (2007) and Samir Kumar Nandy (2013).Throughout the calculations, the parametric values are chosen as $K r=0.2, \eta=0.1, \delta=0.5, \beta=1, \operatorname{Pr}=0.7, S c=0.6, \quad E c=1, \gamma=0.2$, $R=0.1, M=1$.

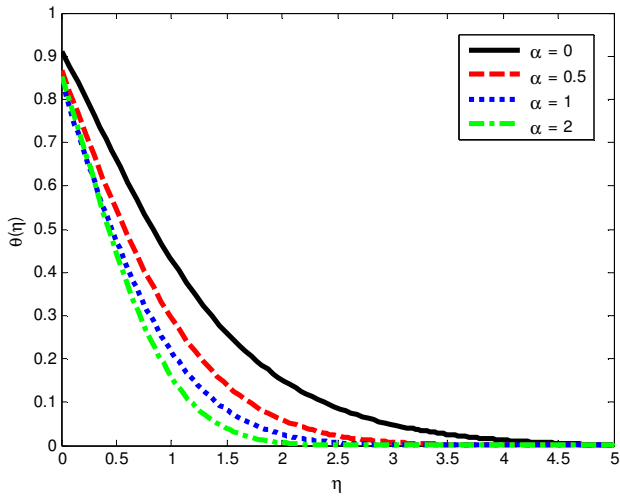

Fig. 3 Effect of $\alpha$ on $\theta(\eta)$.

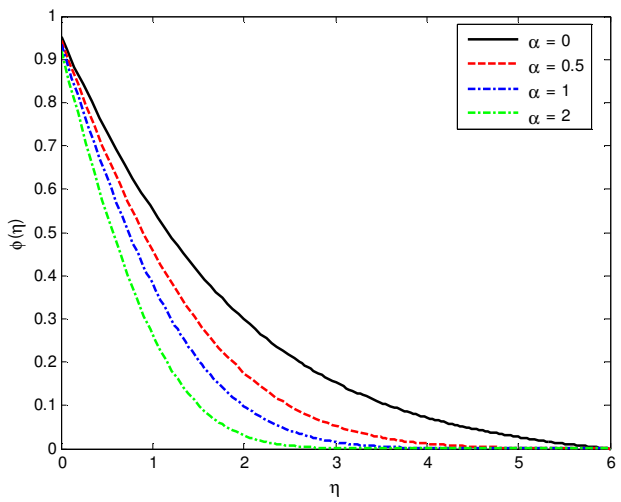

Fig. 4 Effect of $\alpha$ on $\phi(\eta)$.

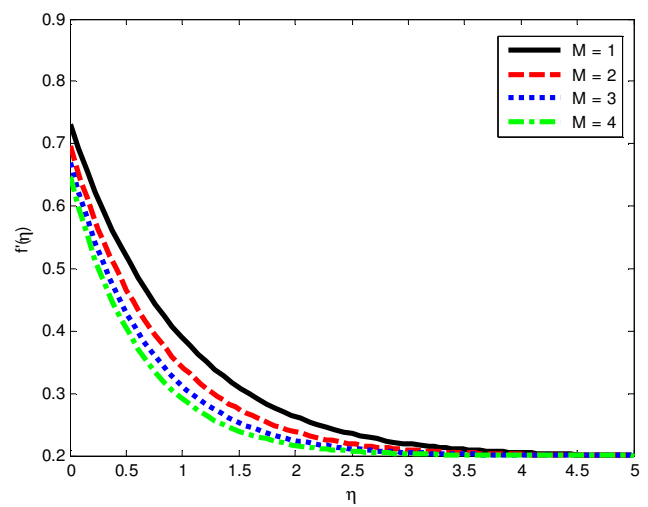

Fig. 5 Effect of $M$ on $f^{\prime}(\eta)$.

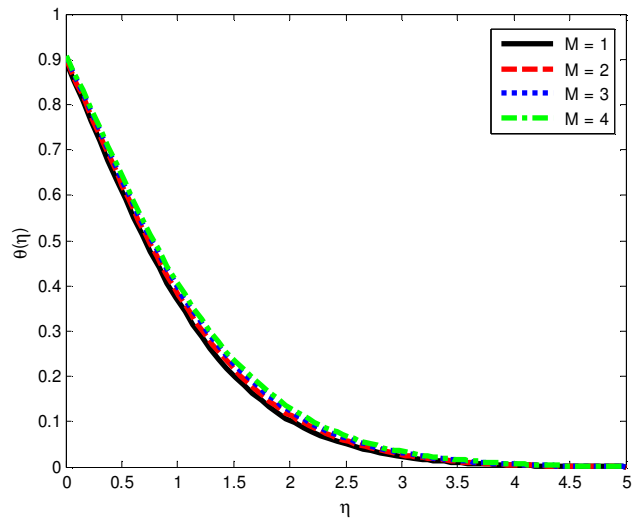

Fig. 6 Effect of $M$ on $\theta(\eta)$.

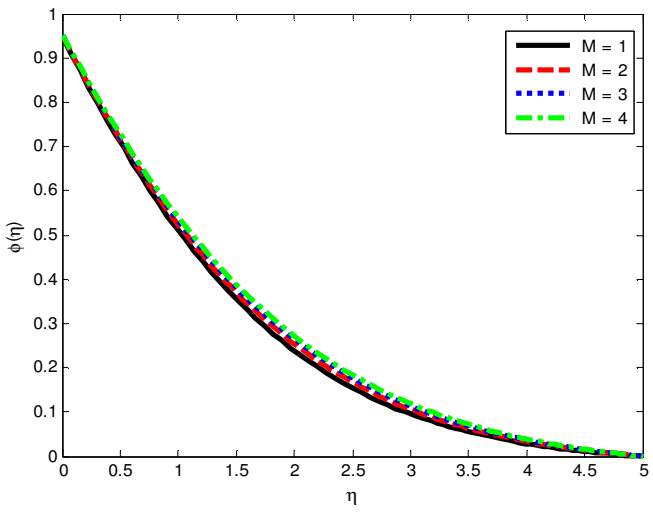

Fig. 7 Effect of $M$ on $\phi(\eta)$.

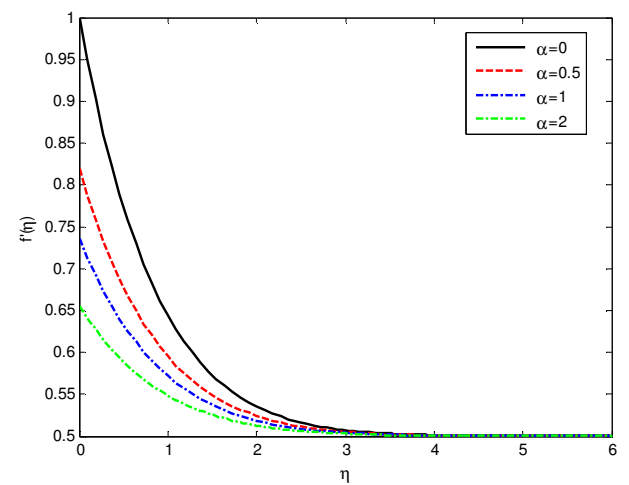

Fig. 8 Effect of $\delta$ on $f^{\prime}(\eta)$.

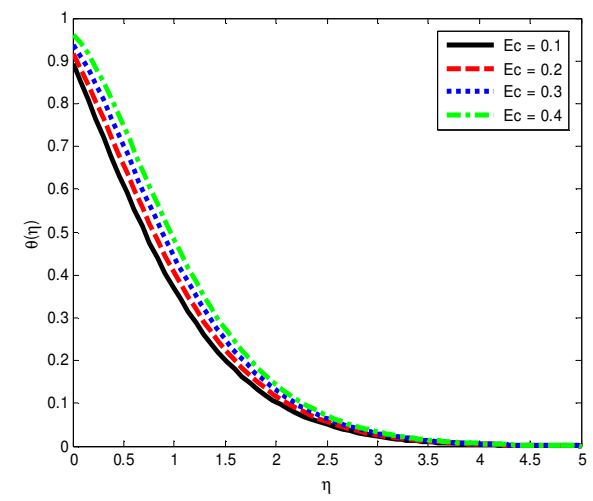

Fig. 9 Effect of $E c$ on $\theta(\eta)$. 


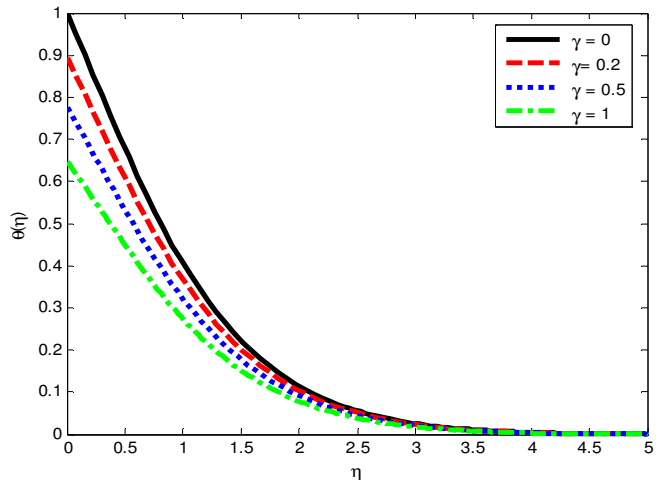

Fig. 10 Effect of $\gamma$ on $\theta(\eta)$.

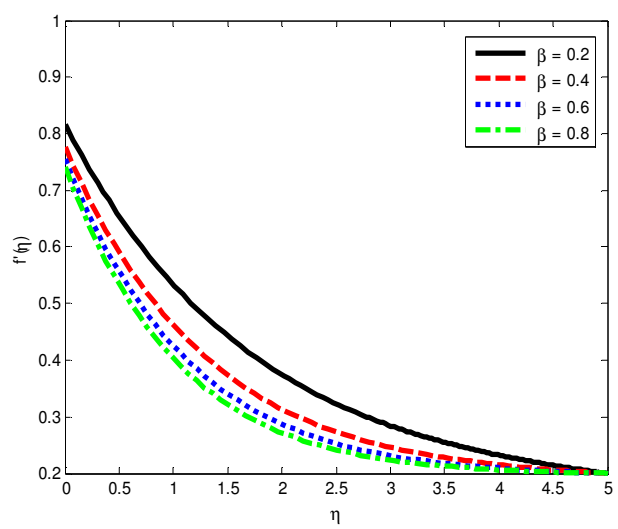

Fig. 11 Effect of $\beta$ on $f^{\prime}(\eta)$.

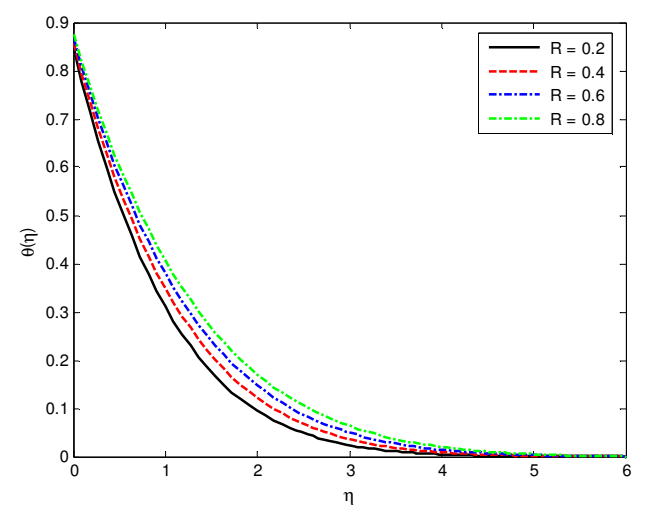

Fig. 12 Effect of $R$ on $\theta(\eta)$.

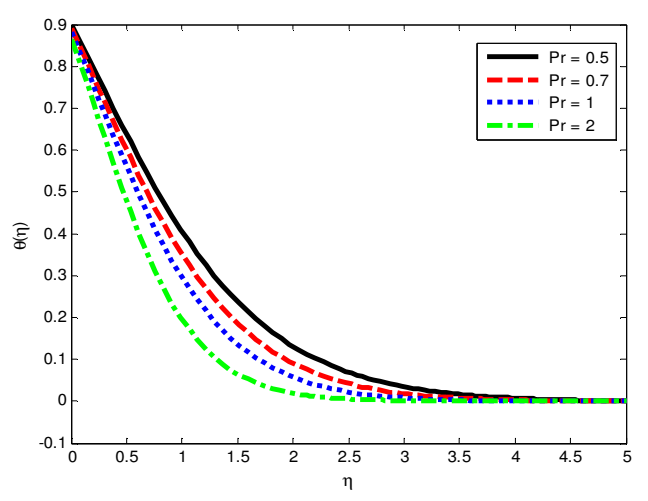

Fig. 13 Effect of $\operatorname{Pr}$ on $\theta(\eta)$.

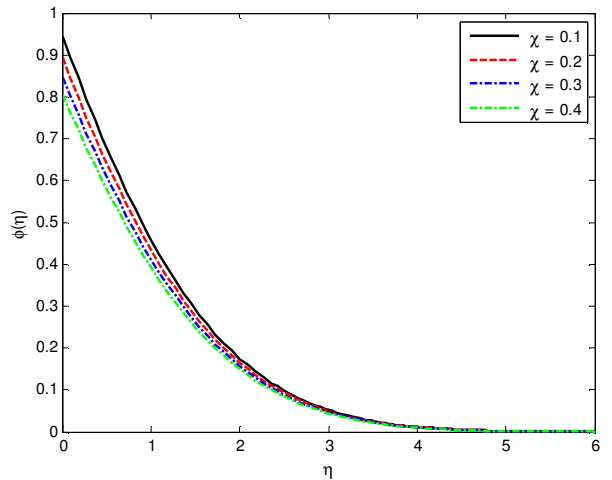

Fig. 14 Effect of $\chi$ on $\phi(\eta)$.

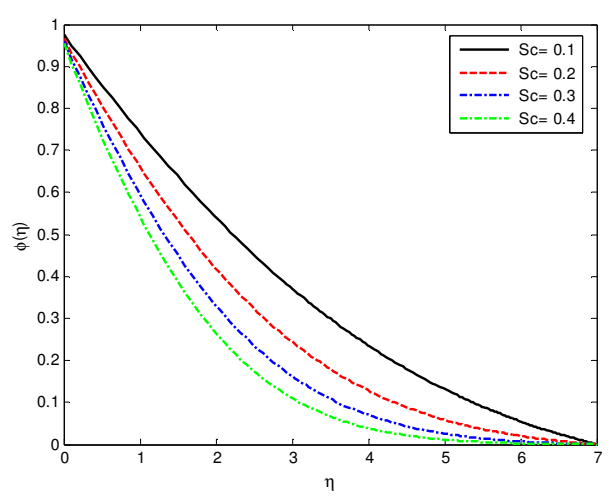

Fig. 15 Effect of $S c$ on $\phi(\eta)$

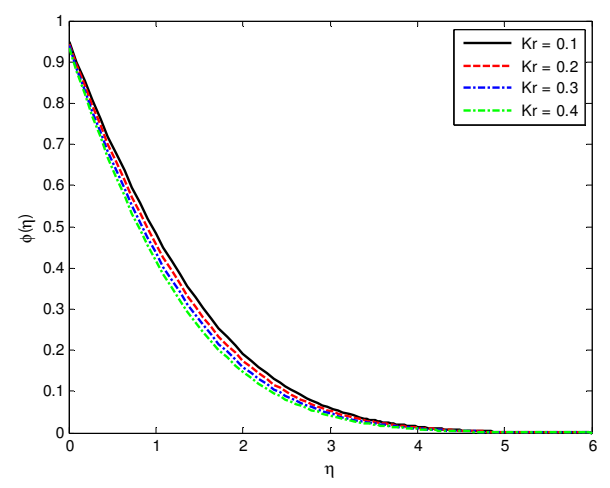

Fig. 16 Effect of $K r$ on $\phi(\eta)$

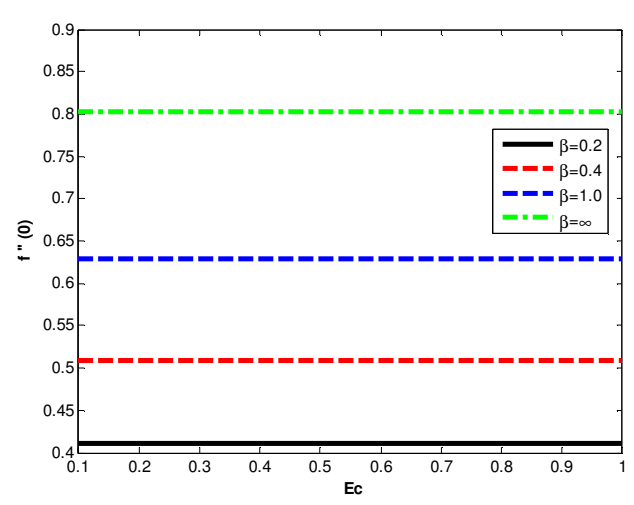

Fig. 17 Effect of $\beta$ on $f^{\prime \prime}(0)$ with $E c$. 


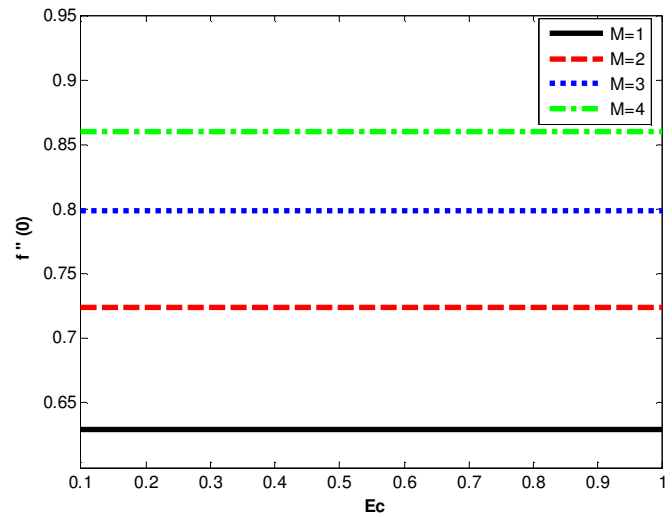

Fig. 18 Effect of $M$ on $f^{\prime \prime}(0)$ with $E c$.

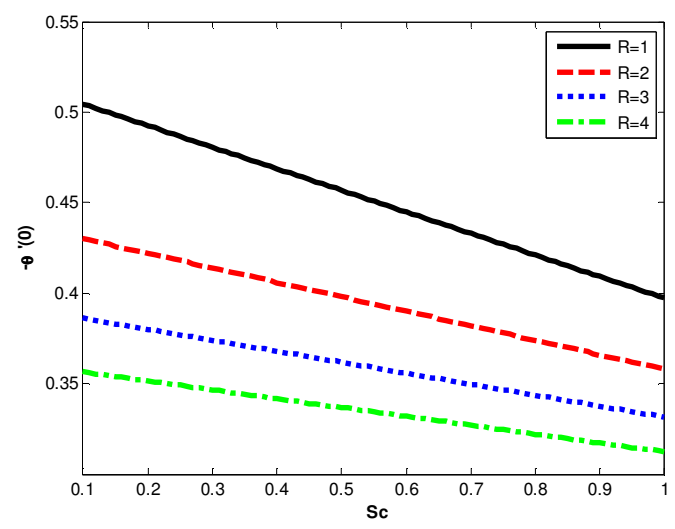

Fig. 19 Effect of $R$ on $-\theta^{\prime}(0)$ with $S c$.

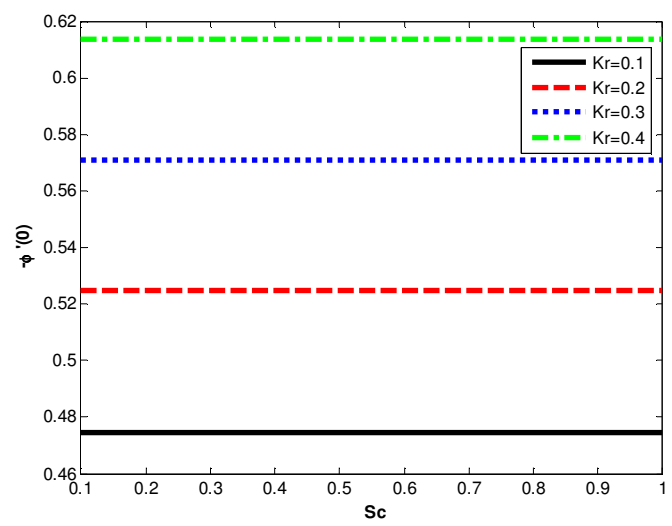

Fig. 20 Effect of $K r$ on $-\phi^{\prime}(0)$ with $S c$.

Table-1: Comparison of the values of $f^{\prime \prime}(0)$ for different values of $\alpha$ with $M=0, \delta=0, \chi=0, S c=0, R=0, K r=0, \beta \rightarrow \infty$.

\begin{tabular}{|c|c|c|c|c|}
\hline$\alpha$ & $\begin{array}{c}\text { Mahapatra and } \\
\text { Gupta(2002) }\end{array}$ & $\begin{array}{c}\text { Ishak et al. } \\
(2007)\end{array}$ & $\begin{array}{c}\text { Samir Kumar } \\
\text { Nandy } \\
(2013)\end{array}$ & $\begin{array}{c}\text { Present } \\
\text { Study }\end{array}$ \\
\hline 0.01 & - & -0.9980 & -0.998024 & -0.999198 \\
\hline 0.10 & -0.9694 & -0.9694 & -0.969386 & -0.969656 \\
\hline 0.20 & -0.9181 & -0.9181 & -0.918107 & -0.918164 \\
\hline 0.50 & -0.6673 & -0.6673 & -0.667264 & -0.667264 \\
\hline 1.0 & 0 & 0 & 0 & 0 \\
\hline 2.0 & 2.0175 & 2.0175 & 2.017507 & 2.017503 \\
\hline
\end{tabular}

Figs. 2-4 illustrate the effect of the ratio parameter on the velocity, temperature and concentration profiles. When the free-stream velocity exceeds the velocity of the stretching sheet, i.e., when $\alpha>1$, the flow velocity increases; however, the boundary layer thickness decreases with an increase in $\alpha$. Furthermore, when the free-stream velocity is greater than the stretching velocity, the velocity approaches the velocity ratio parameter $\alpha$. On the other hand, when the free-stream velocity is less than the velocity of stretching sheet, there is a decrease in velocity of the fluid and hydrodynamic boundary layer thickness. From Figs. 3 and 4 it is observed that both thermal and solutal layer thickness decreases with an increase in velocity ratio parameter $\alpha$. Fig. 5 elucidates that the depreciation of fluid velocity with the rising values of magnetic field parameter. This indicates that the transverse magnetic field opposes the transport phenomena since an increase in $M$ leads to an increase in the Lorentz force, which opposes the transport process. This stronger Lorentz force produces more resistance to the transport. But from Figs. 6 and 7, the opposite phenomenon is observed with an increase in $M$ on temperature and concentration fields respectively. This means the magnetic field works to increase the value of temperature and concentration in the flow field.

Dimensionless velocity profile for various values of velocity slip parameter $\delta$ is shown in Fig. 8. From this figure it is clear that as the values of slip parameter $\delta$ increase, the velocity boundary layer thickness and the surface velocity decrease. This is because under the slip condition, the pulling of the stretching sheet can be only partly transmitted to the fluid. The enhancement of fluid temperature with increasing values of Eckert number $E c$ is shown in Fig. 9. Physically, the Eckert number $E c$ is the relation between flow kinetic energy to heat enthalpy difference. So increase in Eckert number causes enhancement in the kinetic energy. Additionally it is well known fact that temperature is defined as average kinetic energy. Thus alternatively temperature of the fluid rises. It could be analyzed form this graph that fluid temperature increases when Eckert number $E c$ increases.

Fig. 10 presents the variation of fluid temperature with thermal slip parameter $\gamma$. It is seen that on introducing the thermal slip mechanism in the boundary layer, the temperature of the flow is reduced significantly within the boundary layer. Also it is important to note that the effect is prominent within the boundary layer. Fig. 11 shows the effect of Casson parameter $\beta$ on velocity profiles. From this figure, it is seen that the fluid velocity decreases when $\beta$ increases. This is due to the increase of Casson parameter $\beta$, the yield stress $\rho_{y}$ reduces and hence the momentum boundary layer thickness decreases. Physically, it is seen that an increase in $\beta$ leads to an increase in plastic dynamic viscosity that induces resistance in the flow of fluid and decrease in fluid velocity.

Fig. 12 reveals that the fluid temperature increases with an increase in radiation parameter $R$. Physically, increasing the values of the thermal radiation corresponds to an increased dominance of conduction over absorption radiation thus increasing the fluid temperature. Fig. 13 shows the effects of Prandtl number Pr on the heat transfer process. It is observed that the temperature of the fluid is depressed by larger Prandtl number. From a physical point of view, Prandtl number is a dimensionless number approximating the ratio of the momentum diffusivity to the diffusivity. Further, a decrease in the thermal boundary layer with strong Prandtl number is compensated with steeper temperature profiles. The influence of solutal slip parameter $\chi$ on concentration is represented in Fig. 14. It is observed that the concentration of the fluid is higher for small values of solutal slip parameter. This is due to an increase in the mass transfer from the fluid to the porous medium. The variation of dimensionless concentration with Schmidt number $S c$ is shown in Fig. 15. It is seen that the species concentration decrease with the increasing values of $S c$. Physically, Schmidt number is the ratio of the viscous diffusion rate to mass diffusion rate, the increase in $S c$ results in an increase of the viscous diffusion rate which reduces the concentration. Fig. 16 demonstrates the effect of distractive chemical reaction parameter 
$(K r>0)$ on concentration field. It is seen that the destructive chemical reaction within the boundary layer has the tendency to decrease the solute concentration. This is accompanied by a slight decrease in the solutal boundary layer thickness and the negative wall slope of the concentration profile. For non-destructive chemical reactions, exactly the opposite effect is predicted.

Fig. 17 exhibits the nature of skin friction coefficient $f^{\prime \prime}(0)$ for different values of Casson parameter $\beta$ and magnetic field parameter $M$ against Eckert number $E c$. As expected, the skin friction increases as the Casson parameter increases. Physically, the negative sign of $f^{\prime \prime}(0)$ implies that surface exerts a dragging force on the fluid and positive sign implies the opposite. The effect of magnetic field parameter $M$ against Eckert number $E c$ on skin friction coefficient is shown in Fig. 18. It is seen that the skin friction increase with an increase in $M$.Physically, the application of magnetic field perpendicular to the fluid flow produces a drag force which tends to retard the fluid flow velocity, thus increasing the skin friction coefficient.

Fig. 19 displays the nature of heat transfer coefficient $-\theta^{\prime}(0)$ against Schmidt number $S c$ for various values of radiation parameter $R$. It is clear that the heat transfer rate decreases with an increase in $R$. The rate of mass transfer coefficient against Schmidt number $S c$ for different values of chemical reaction parameter $K r$ is shown in Fig. 20. It is observed that the mass transfer rate increase with an increase in $K r$. We compared our results with the existing results of Mahapatra and Gupta et al. (2002), Ishak et al. (2007) and Samir Kumar Nandy (2013), as shown in Table1, for various values of velocity ratio parameter $\alpha$ on skin friction coefficient $f^{\prime \prime}(0)$. The table depicts an excellent agreement between our result and that of others as mentioned above which in turn gives a confidence that the numerical results obtained by us are accurate.

\section{CONCLUSIONS}

In the present study, we have considered thermal radiation and chemical reaction effects on MHD stagnation point flow of an electrically conducting viscous incompressible Casson fluid through a permeable stretching sheet with multiple slips in the presence of viscous and Joule dissipation. Using Matlab bvp4c package, we developed the series solutions of governing problem.

Following conclusions are drawn from the present study.

1. The momentum boundary layer thickness decreases with an increase in the Casson parameter $\beta$, but the Skin friction coefficient increases with $\beta$.

2. Thermal boundary layer thickness decreases with an increase in both velocity ratio parameter A and Prandtl number Pr.

3. The heat transfer rate decreases with Radiation parameter $R$.

4. The velocity, temperature and concentration decrease with an increase of velocity slip, thermal slip and solutal slip parameters.

5. The increase in chemical reaction parameter decreases the dimensionless concentration in the concentration boundary layer. Thus mass transfer rate increases with chemical reaction parameter $\gamma$.

\section{REFERENCES}

Abo-Eldahab, E.M. and El Aziz, M.A., 2005, "Viscous Dissipation and Joule Heating Effects on MHD-Free Convection from a Vertical Plate with Power-Law Variation in Surface Temperature in the Presence of Hall and Ios-Slips Currents," Applied Mathematical Modelling, 29, 579-595. https://doi.org/10.1016/i.apm.2004.10.005.

Alim, M.A., Alam, M.D., and Mamun, A., 2007, "Joule Heating Effect on the Coupling of Conduction with Magnetohydrodynamic Free Convection Flow from a Vertical Flat Plate," Non-linear Analysis: Modelling and Control, 12, 307-316.

https://dx.doi.org/10.1.1.523.7176.

Al-sudais, N.S., 2012, “Thermal Radiation Effects on MHD Fluid Flow near Stagnation Point of Linear Stretching Sheet with Variable Thermal Conductivity," International Mathematical Forum, 51, 2525-2544. https://dx.doi.org/10.1.1.674.8152.

Animasaun, I.L., Adebile, E.A. and Fagbade, A.I, 2016, "Casson Fluid Flow with Variable Thermo-physical Property along Exponentially Stretching Sheet with Suction and Exponentially Decaying Internal Heat Generation using the Homotopy Analysis Method," Journal of the Nigerian Mathematical Society, 35, 1-17. https://doi.org/10.1016/j.jnnms.2015.02.001.

Bhattacharyya, K., 2013, "Boundary Layer Stagnation Point Flow of Casson Fluid and Heat Transfer towards a Shrinking/Stretching Sheet," Frontiers in Heat and Mass Transfer, 4, 1-9. https://doi.org/10.5098/hmt.v4.2.3003.

Bhattacharyya, K., 2013, "MHD Stagnation Point Flow of Casson Fluid and Heat Transfer over a Stretching Sheet with Thermal Radiation," Journal of Thermodynamics, 2013, 1-9. https://dx.doi.org/10.1155/2013/169674.

Bhattacharyya, K., Mukhopadhyay, S. and Layek, G.C., 2011, "MHD boundary layer slip flow and heat transfer over a flat plate," Chinese Physics Letter, 28, 1-4. https://dx.doi.org/10.1088/0256-307X/28/2/024701.

Bird, R.B., Stewart, W.E. and Lightfoot, E.N., 1960, “Transport Phenomena," Wiley publishers, New York., 94, 104-105. https://dx.doi.org/10.1002/14356007.b01_04.

Brewster, M.Q., 1972, "Thermal Radiative Transfer Properties," John Wiley and Sons.

Casson, N., 1959, "Rheology of Dispersed Systems," Pergamon Press, Oxford, 84-104.

Dash, R.K., Mehta, K.N. and Jayaraman, G., 1996 "Casson Fluid Flow in a Pipe Filled with a Homogeneous Porous Medium," International Journal of Engineering Science, 34, 1145-1156. https://dx.doi.org/10.1016/0020-7225(96)00012-2.

Dorier, C., and Tichy, J., 1992 "Behaviour of a Bingham-like Viscous Fluid in Lubrication Flows," Journal of Non-Newtonian Fluid Mechanics, 45, 291-310. https://dx.doi.org/10.1016/0377-0257(92)80065-6.

Gireesha, B.J., Mahanthesh. B. and Rashidi, M. M., 2015, "MHD Boundary Layer Heat and Mass Transfer of a Chemically Reacting Casson Fluid over a Permeable Stretching Surface with NonUniform Heat Source/Sink," International Journal of Industrial Mathematics, 7, 247-260.

Hayat, T., Shehzad, S.A., Alsaedi, A. and Alhothuali, M.S., 2012, "Mixed Convection Stagnation Point Flow of Casson Fluid with Convective Boundary Conditions," Chinese Physics Letters, 29, $1147041-4$

https://dx.doi.org/10.1088/0256-307X/29/11/114704 
Hiemenz, K., 1911, "Die Grenzchicht an einem inden Gleichformingen Flussigkeitsstrom eingetauchten geraden Kreiszylinder," Dingler's Polytechnisches Journal, 326, 321-410. http://dingler.culture.hu-berlin.de/article/pj326/ar326114.

Ishak, A., Jafar, K., Nazar, R. and Pop, I., 2009, “MHD Stagnation Point Flow towards a Stretching Sheet," Physica A: Statistical Mechanics and its Applications, 388, 3377-3383.

https://dx.doi.org/10.1016/j.physa.2009.05.026.

Ishak, A., Nazar, R., Arifin, N.M. and Pop, I., 2007, "Mixed Convection of the Stagnation Point Flow towards a Stretching Vertical Permeable Sheet," Malaysian Journal of Mathematical Sciences, 2, 217-226.

Joye, D.D., 1998, "Hazardous and Industrial Wastes: Proceedings of the Thirty -Atlantic Industrial and Hazardous Waste Conference," Technomic Public- Corporation, Lancaster,

PA/Basel, 567-575.

Kirsanov, E.A. and Remizov, S.V., 1999, "Application of the Casson Model to Thixotropic Waxy Crude Oil," Rheologica Acta, 38, 172-176.

https://dx.doi.org/10.1007/s003970050166.

Mahapatra, T.R. and Gupta, A.S., 2002, "Heat Transfer in Stagnation Point Flow towards a Stretching Sheet," Heat and Mass Transfer, 38, 517-521.

https://dx.doi.org/10.1007/s002310100215.

Medikare, M., Sucharitha.J. and Kishore Kumar, C., 2016 "MHD Stagnation Point Flow of a Casson Fluid over a Nonlinearly Stretching Sheet with Viscous Dissipation," American Journal of Computational Mathematics, 6, 37-48.

https://dx.doi.org/10.4236/ajcm.2016.61005.

Mohammad R. Hajmohammadi and Nourazar, S.S., 2014, "Conjugate Forced Convection Heat Transfer from a Heated Flat Plate of Finite Thickness and Temperature - Dependent Thermal Conductivity," Heat Transfer Engineering, 35, 863-874.

https://dx.doi.org/10.1080/01457632.2014.852896.

Mustafa, M., Hayat, T., Pop, I. and Hendi, A., 2012, "Stagnation Point Flow and Heat Transfer of a Casson Fluid Towards a Stretching Sheet," Z Naturforsch, 67, 70-76. https://dx.doi.org/10.5560/zna.2011-0057.

Nadeem, S., Haq, R.U. and Lee, C., 2012, "The Magneto hydrodynamic (MHD) Boundary Layer Flow of a Casson Fluid over an Exponentially Penetrable Shrinking Sheet," Scientia Iranica, 19, 1550-1553.

https://dx.doi.org/10.1016/j.scient.2012.10.021.

Nandy, S.K., 2013, “Analytical Solution of MHD Stagnation Point Flow and Heat Transfer of Casson Fluid over a Stretching Sheet with Partial Slip," ISRN Thermodynamics, 2013, 1-9. https://dx.doi.org/10.1155/2013/108264.

Prasanna kumara, B.C., Krishnamurthy, M.R., Gireesha, B.J. and Rama Subba Reddy, G., 2016, "Effect of Multiple Slips and Thermal Radiation on MHD Flow of Jeffery Nanofluid with Heat Transfer," Journal of nanofluids, 5, 82-93. https://dx.doi.org/10.1166/jon.2016.1198.

Rajagopal, K.R., 1980, "Viscometric Flows of Third Grade Fluids," Mechanics Research Communications, 7, 21-25.

Rajagopal, K.R., Na. T.Y. and Gupta, A.S., 1984, "Flow of a Viscoelastic Fluid over a Stretching Sheet," Rheologica Acta, 23, 213-215. https://dx.doi.org/10.1007/BF01332078.

Ramesh, G.K., Gireesha, B.J. and Bagewadi, C. S., 2014 "Stagnation Point Flow of a MHD Dusty Fluid towards a Stretching Sheet with Radiation," Afrika Matematika, 25, 237249 .

https://dx.doi.org/10.1007/s13370-012-0114-6.

Ramesh, G.K., Prasanna kumara, B.C., Gireesha, B.J., and Rashidi, M.M., 2016, "Casson Fluid Flow near the Stagnation Point over a Stretching Sheet with Variable Thickness and Radiation," Journal of Applied Fluid Mechanics, 9, 1115-1122. https://dx.doi.org/10.18869/acadpub.jafm.68.228.24584.

Turkyilmazoglu, M., 2011, "Multiple Solutions of Heat and Mass Transfer of MHD Slip Flow for the Viscoelastic Fluid over a Stretching Sheet," International Journal of Thermal Sciences, 50, 2264-2276.

https://dx.doi.org/10.1016/j.ijthermalsci.2011.05.014.

Wilkinson, W.L., 1970, "The Drainage of a Maxwell Liquid Down a Vertical Plate," The Chemical engineering Journal, 1, 255-257. https://dx.doi.org/10.1016/0300-9467(70)80008-9.

Yih, K.A., 2002, "Viscous and Joule Heating Effects on NonDarcy MHD Natural Convection Flow over a Permeable Sphere in Porous Media with Internal Heat Generation," International Communications in Heat and Mass Transfer, 27, 591-600. https://dx.doi.org/10.1016/S0735-1933(00)00141-X. 